\title{
Determination of neutron fracture functions from a global QCD analysis of the leading neutron production at HERA
}

\author{
Samira Shoeibi, ${ }^{1, *}$ Hamzeh Khanpour, ${ }^{2,3, \dagger}$ F. Taghavi-Shahri, ${ }^{1, \star}$ and Kurosh Javidan ${ }^{1, \S}$ \\ ${ }^{1}$ Department of Physics, Ferdowsi University of Mashhad, P.O.Box 1436 Mashhad, Iran \\ ${ }^{2}$ Department of Physics, University of Science and Technology of Mazandaran, \\ P.O.Box 48518-78195 Behshahr, Iran \\ ${ }^{3}$ School of Particles and Accelerators, Institute for Research in Fundamental Sciences (IPM), \\ P.O.Box 19395-5531 Tehran, Iran
}

(Received 13 March 2017; published 10 April 2017)

\begin{abstract}
In this article, we present our global QCD analysis of leading neutron production in deep inelastic scattering at $\mathrm{H} 1$ and ZEUS collaborations. The analysis is performed in the framework of a perturbative QCD description for semi-inclusive processes, which is based on the fracture functions approach. Modeling the nonperturbative part of the fragmentation process at the input scale $\mathrm{Q}_{0}^{2}$, we analyze the $\mathrm{Q}^{2}$ dependence of the leading neutron structure functions and obtain the neutron fracture functions (neutron FFs) from next-toleading order global QCD fit to data. We have also performed a careful estimation of the uncertainties using the "Hessian method" for the neutron FFs and corresponding observables originating from experimental errors. The predictions based on the obtained neutron FFs are in good agreement with all data analyzed, at small and large longitudinal momentum fraction $x_{L}$ as well as the scaled fractional momentum variable $\beta$.
\end{abstract}

DOI: 10.1103/PhysRevD.95.074011

\section{INTRODUCTION}

Over the past decade, our knowledge of the quark and gluon substructure of the nucleon has been extensively improved due to the high-energy scattering data from fixed target experiments, the precise data from the electron-proton collider HERA [1-8], the data from high energy protonproton scattering at the Tevatron [9-18], and up-to-date data from LHC [19-30]. Deep inelastic scattering (DIS) data as well as data from hadron colliders has been successfully used in many global QCD analyses to extract the unpolarized parton distribution functions (PDFs) [31-37], polarized PDFs [38-44], nuclear PDFs [45-50], and related studies [51-71]. Beside the mentioned data sets, the production of leading neutron and proton in deep inelastic scattering (DIS) opens a new window for the theory of strong interaction in the soft region and provides a probe of the relationship between QCD of quarks and gluons and the strong interaction of hadrons [72,73]. Consequently, in the framework of perturbative QCD (pQCD), the study of leading-baryon production represents an important field of investigation. In the leading-baryon productions, $e p \rightarrow e^{\prime} B X$, the energetic neutron or proton which are produced in the fragmentation of the proton remnant, carry a large fraction $x_{L}$ of the longitudinal momentum of the incoming proton [72-82]. These events are measured at a small polar angle with respect to the collision axis.

\footnotetext{
*Samira.Shoeibimohsenabadi@mail.um.ac.ir

†Hamzeh.Khanpour@mail.ipm.ir

Taghavishahri@um.ac.ir

§Javidan@um.ac.ir
}

However, due to the difficulty of detecting the leadingbaryon in high energy physics experiment, the data available are scarce. More recently, the $\mathrm{H} 1$ and ZEUS collaborations at HERA have measured events, in which a neutron is produced in the forward region, obtain sizable contributions of leading neutrons to the DIS cross sections, $\sim 8 \%-10 \%[72,73]$. These kinds of processes open a new window to study hard processes in a new kinematical region to obtain information on soft quantum chromodynamics (QCD) dynamics. In that case, the measurements of leading-baryon structure functions can be used as a test of new aspects of QCD. Along with these experimental developments, the fracture functions approach has been developed in the framework of perturbative QCD in order to deal with such a kind of forward processes [83-85].

Fracture functions provide a QCD-based description of semi-inclusive DIS in the target fragmentation region. The formalism of fracture functions, where the leading particles production is described in terms of structure functions of the fragmented nucleon, has been successfully used to describe forward neutron data from the $\mathrm{H} 1$ and ZEUS collaborations [86-88]. As for the DIS structure function, QCD can not predicted the shape of fracture functions. As for parton distribution functions (PDFs), the nonperturbative neutron fracture function (neutron FFs) can be parametrized at a given initial scale $\mathrm{Q}_{0}^{2}$. Fracture functions, the probabilities of finding a parton and a hadron in the target, can be related to the parton distributions of the object exchanged between the initial and final states. In the production of the leading neutron in the target fragmentation region, this object is $\pi^{+}$in the $e p \rightarrow e n X$ process. 
In the target fragmentation region, the corresponding cross sections are expressed as a convolution of the fracture functions, $M_{h / p}$, with the point like partonic cross sections. In this paper, we present the results of our QCD global analysis of recent and up-to-date experimental data for the production of neutrons in the forward direction in DIS. As we mentioned, the results obtained in this analysis, is in the framework of fracture functions by modeling the neutron FFs at the input scale, $\mathrm{Q}_{0}^{2}$. We propose a standard parametric form for the neutron FFs at a given initial scale $\mathrm{Q}_{0}^{2}=1 \mathrm{GeV}^{2}$ and obtain their parameters by next-toleading order (NLO) global QCD fit to forward neutron production data measured by $\mathrm{H} 1$ and ZEUS collaborations at HERA. We find that our theory predictions are in satisfactory agreement with all data analyzed.

The outline of the present paper is the following. First, in Sec. II, we present the theoretical settings of the analysis. The details of the fitting methodology applied in this work and the functional forms used to extract neutron FFs are presented in Sec. III. The details of the forward neutron production data from $\mathrm{H} 1$ and ZEUS collaborations are discussed in Sec. IV. Section V provides the method of the $\chi^{2}$ minimization, uncertainties estimation, and error calculations. The results of present NLO neutron FFs fits and a detailed comparison with available observables are discussed in Sec. VI. In Sec. VII, we briefly discuss the present and upcoming experimental data on the production of leading baryons at LHC and at the Jefferson Lab. Finally, Sec. VIII contains the summary and conclusions.

\section{THEORETICAL FRAMEWORK}

We can now specify the theory settings used for the neutron FFs fits presented in this work. We will restrict ourselves to a brief summary of the theoretical framework relevant for our global QCD analysis of leading neutron structure functions in which we closely follow Ref. [86].

We use the NLO theory with $\alpha_{s}\left(M_{Z}^{2}\right)=0.1184$ in a variable flavor number scheme (VFNS) with charm and bottom masses of $m_{c}=1.41$ and $m_{b}=4.50 \mathrm{GeV}$. In order to describe the hard scattering DIS process, we use the usual kinematic variables $x, \mathrm{Q}^{2}, y$, which are defined as

$$
Q^{2}=-q^{2}, \quad x=\frac{Q^{2}}{2 p \cdot q}, \quad y=\frac{p \cdot q}{p \cdot k},
$$

where in the DIS process, $p$ is the four-momenta of the incident proton, $k$ is the four-momenta of the incident positron, and $q$ is the four-momenta of the virtual photon. The fourfold differential cross section to describe the baryon production processes $e p \rightarrow e^{\prime} B X$ can be obtained by the semi-inclusive leading-baryon transverse and longitudinal structure functions, $F_{2}^{\mathrm{LB}(4)}$ and $F_{L}^{\mathrm{LB}(4)}$, which are defined as [72,73]

$$
\begin{aligned}
\frac{d^{4} \sigma\left(e p \rightarrow e^{\prime} B X\right)}{d \beta d Q^{2} d x_{L} d t}= & \frac{4 \pi \alpha^{2}}{\beta Q^{4}}\left(1-y+\frac{y^{2}}{2}\right) F_{2}^{\mathrm{LB}(4)}\left(\beta, Q^{2}, x_{L}, t\right) \\
& +F_{L}^{\mathrm{LB}(4)}\left(\beta, Q^{2}, x_{L}, t\right) .
\end{aligned}
$$

The longitudinal momentum fraction $x_{L}$ and the scaled fractional momentum variable $\beta$ are defined by

$$
x_{L} \simeq \frac{E_{B}}{E_{p}}, \quad \beta=\frac{x}{1-x_{L}},
$$

where $x$ is the Bjorken variable, $E_{p}$ is the proton beam energy, and $E_{B}$ is the energy of final-state baryon. In Eq. (2), $t$ is the squared four-momentum transfer between the incident proton and the final state neutron. The $t$ integrated differential cross section can be obtained by

$$
\begin{aligned}
\frac{d^{3} \sigma\left(e p \rightarrow e^{\prime} B X\right)}{d \beta d Q^{2} d x_{L}}= & \int_{t_{0}}^{t_{\min }} \frac{d^{4} \sigma\left(e p \rightarrow e^{\prime} B X\right)}{d \beta d Q^{2} d x_{L} d t} d t \\
= & \frac{4 \pi \alpha^{2}}{\beta Q^{4}}\left(1-y+\frac{y^{2}}{2}\right) F_{2}^{\mathrm{LB}(3)}\left(\beta, Q^{2}, x_{L}\right) \\
& +F_{L}^{\mathrm{LB}(3)}\left(\beta, Q^{2}, x_{L}\right)
\end{aligned}
$$

where the integration limits are

$$
\begin{aligned}
t_{\min } & =-\left(1-x_{L}\right)\left(\frac{m_{N}^{2}}{x_{L}}-m_{p}^{2}\right), \\
t_{0} & =t_{\min }-\frac{\left(p_{T}^{\max }\right)^{2}}{x_{L}} .
\end{aligned}
$$

$m_{N}$ is the mass of the final-state baryon, $m_{p}$ is the proton mass, and $p_{T}^{\max }$ is the upper limit of the neutron transverse momentum used for the $F_{2}^{\mathrm{LB}(3)}$ measurement. For the semi-inclusive processes which have a final-state proton and neutron, the structure function $F_{2, L}^{\mathrm{LB}(3)}$, is denoted by $F_{2, L}^{\mathrm{LP}(3)}$ and $F_{2, L}^{\mathrm{LN}(3)}$, respectively. In this paper, which corresponds to a QCD analysis of a forward neutron production, we define the reduced $e^{+} p$ cross section $\sigma_{r}^{\mathrm{LN}(3)}$ in terms of the leading neutron transverse $F_{2}^{\mathrm{LN}(3)}$ and the longitudinal structure functions $F_{L}^{\mathrm{LN}(3)}$ as $[72,73]$

$$
\begin{aligned}
\sigma_{r}^{L N(3)}= & F_{2}^{L N(3)}\left(\beta, Q^{2}, x_{L}\right) \\
& -\frac{y^{2}}{1+(1-y)^{2}} F_{L}^{L N(3)}\left(\beta, Q^{2}, x_{L}\right) .
\end{aligned}
$$

It is noteworthy to mention here that the leading neutron structure functions in the above equations can be written in terms of neutron FFs and hard-scattering coefficients [86]. The Wilson coefficient functions are the same as in a fully inclusive DIS [89]. 
The well-known DGLAP evolution equations [90-93], which are a set of an integrodifferential equations, can be used to evolve the polarized and unpolarized parton distributions functions to an arbitrary energy scale, $Q^{2}$. The solutions of these evolution equations will provide us the valance, gluon, and sea quark distributions inside the nucleon. These equations can be used widely as fundamental tools to extract the deep inelastic scattering (DIS) structure functions of a proton, neutron, and deuteron, which enrich our current information about the structure of the hadrons. Since the scale dependence of the cross section in the forward particle production in DIS can be calculated within perturbative quantum chromodynamics (pQCD) [83]; consequently, the neutron fracture functions also obey the standard DGLAP evolution equations [86,94].

In Refs. [87,94-98], it has been shown that, in the phenomenological level, the fracture functions well reproduce the leading proton data; thus, one can use the common perturbative QCD approach to these particular classes of semi-inclusive processes. So, like for the case of parton distributions functions (PDFs), one can use a phenomenological model to describe a forward neutron production and extract the neutron FFs from the QCD fit to the data $[84,86]$. The evolution equations of neutron FFs are easily obtained by the DGLAP evolution equations [83] as

$$
\begin{aligned}
Q^{2} & \frac{\partial M_{\Sigma / P}^{B}\left(\beta, Q^{2}, x_{L}\right)}{\partial Q^{2}} \\
& =\frac{\alpha_{s}\left(Q^{2}\right)}{2 \pi} \int_{\beta}^{1} \frac{d u}{u} P_{\Sigma}^{j}(u) M_{\Sigma / P}^{B}\left(\frac{\beta}{u}, Q^{2}, x_{L}\right), \\
Q^{2} & \frac{\partial M_{g / P}^{B}\left(\beta, Q^{2}, x_{L}\right)}{\partial Q^{2}} \\
& =\frac{\alpha_{s}\left(Q^{2}\right)}{2 \pi} \int_{\beta}^{1} \frac{d u}{u} P_{g}^{j}(u) M_{g / P}^{B}\left(\frac{\beta}{u}, Q^{2}, x_{L}\right),
\end{aligned}
$$

where $M_{\Sigma / P}^{B}\left(\beta, Q^{2}, x_{L}\right)$ and $M_{g / P}^{B}\left(\beta, Q^{2}, x_{L}\right)$ correspond to the singlet and gluon distributions, respectively [84]. These nonperturbative distributions, which hereafter are indicated by "neutron FFs", need to be parametrized at an input scale, $\mathrm{Q}_{0}^{2}$. Their evolution to a higher scale, $Q^{2}>Q_{0}^{2}$, can be described by using the evolution equation given above. $P_{\Sigma}$ and $P_{g}$ in Eq. (7) are the common NLO contributions to the splitting functions governing the evolution of unpolarized singlet and nonsinglet combinations of quark densities in perturbative QCD. Splitting functions are perturbatively calculable as a power expansion in the strong coupling constant $\alpha_{s}$. The splitting functions $P_{\Sigma}$ and $P_{g}$ in Eq. (7) are the same as in fully inclusive DIS [99-103].

In the next sections, we give a detailed account of the first global analysis of neutron FFs performed in this study, which in the following will be referred to as "SKTJ17". We first discuss in detail the parametrization of neutron FFs, and then we will present data selection and the determination of the best fit, which we compare to the fitted data. We then focus on the studies of uncertainties using the standard Hessian error matrix approach.

\section{NLO QCD ANALYSIS OF NEUTRON FFS AND PARAMETRIZATION}

In order to obtain a parametrization for the neutron FFs, $\beta M_{i / P}^{N}\left(\beta, Q_{0}^{2}, x_{L}\right)$ with $i=\Sigma$ and $g$, at a given initial scale $Q_{0}^{2}$, we select a relatively simple functional dependence in the variables $\beta$ and $x_{L}$ with enough flexibility as to reproduce the data accurately. We assume the following general initial functional form at $\mathrm{Q}_{0}^{2}=1 \mathrm{GeV}^{2}$ :

$\beta M_{\Sigma / P}^{N}\left(\beta, Q_{0}^{2}, x_{L}\right)=\mathcal{A}_{q}\left(x_{L}\right) \beta^{a_{q}}(1-\beta)^{b_{q}}\left(1+c_{q} \beta\right)$,

$\beta M_{g / P}^{N}\left(\beta, Q_{0}^{2}, x_{L}\right)=\mathcal{A}_{g}\left(x_{L}\right) \beta^{a_{g}}(1-\beta)^{b_{g}}\left(1+c_{g} \beta\right)$,

where $\mathcal{A}_{q}\left(x_{L}\right)$ and $\mathcal{A}_{g}\left(x_{L}\right)$ are defined as

$$
\begin{aligned}
& \mathcal{A}_{q}\left(x_{L}\right)=\mathcal{N}_{q} x_{L}^{A_{q}}\left(1-x_{L}\right)^{B_{q}}\left(1+C_{q} x_{L}^{D_{q}}\right), \\
& \mathcal{A}_{g}\left(x_{L}\right)=\mathcal{N}_{g} x_{L}^{A_{g}}\left(1-x_{L}\right)^{B_{g}}\left(1+C_{g} x_{L}^{D_{g}}\right) .
\end{aligned}
$$

The label of $\Sigma / P$ and $g / P$ corresponds to the singlet and gluon distributions, respectively. The $x_{L}$ dependence of the neutron FFs is encoded in $\mathcal{A}_{i}\left(x_{L}\right)$. Since the present leading neutron data are not yet sufficient to distinguish $q(=u, d, s)$ from $\bar{q}(=\bar{u}, \bar{d}, \bar{s})$, we assume symmetric sea distributions throughout. We will show that these kinds of parametrizations give relatively good initial approximations to the description of the $\mathrm{H} 1$ and ZEUS leading neutron data sets $[72,73]$; however, their survival seems unlikely in a more precise analysis. The available forward neutron production data are not accurate enough to determine all the shape parameters with sufficient accuracy. Equation (8) includes 18 free parameters in total in which we further reduce the number of free parameters in the final minimization.

The parameters $\left\{p_{i}\right\}$ representing our best global QCD fit of neutron FFs in Eq. (8), henceforth denoted as SKTJ17, are given in Table III. A few additional remarks will be presented in Sec VI. As we mentioned, the currently available leading neutron data do not fully constrain the entire $\beta$ and $x_{L}$ dependence of $\beta M_{\Sigma / P}^{N}$ and $\beta M_{g / P}^{N}$ imposed in Eq. (8). Consequently, we are forced to make some restrictions on the parameter space $\left\{p_{i}\right\}$. We will return to this issue in a separate section.

Rather than also determining the strong coupling $\alpha_{s}\left(Q_{0}^{2}\right)$ in the global QCD fit along with the neutron FFs parameters, we fixed the $\alpha_{s}\left(M_{Z}^{2}\right)$ value close to the updated Particle Data Group (PDG) average. The scale dependence of $\alpha_{s}$ is normally computed by numerically solving its renormalization group equation at a next-to-leading order accuracy. For the evolution, we take $\alpha_{s}\left(M_{Z}^{2}\right)=0.1184$ 
$[104,105]$, and we choose to work in the variable flavor number scheme (VFNS), where charm and bottom quark distributions are radiatively generated from their corresponding thresholds [34,35]. In the present analysis, all quarks are treated as massless, and we fixed the heavy quark masses at $m_{c}=1.41 \mathrm{GeV}, m_{b}=4.50 \mathrm{GeV}$, and $m_{b}=175.0 \mathrm{GeV}$. Our choice for the VFNS scheme is due to the fact that for all presently available leading neutron observables, heavy quarks play a negligible role. The scale evolution equations for the neutron FFs are solved in $x$ space at next-to-leading order. Likewise, all leading neutron observables used in our QCD fit are computed consistently at a next-to-leading order accuracy in the $\overline{\mathrm{MS}}$ factorization scheme.

\section{LEADING NEUTRON PRODUCTION DATA}

Our first physics objective is to establish the set of neutron FFs that gives the optimum theoretical description of the available hard scattering leading neutron production data. In this section, we will present the data sets used in the present analysis. The data sets that we will use is the following: The $\mathrm{H} 1$ data on the leading neutron production in DIS scattering [72] as well as the data from the leading neutron production in an $e^{+} p$ collision from the ZEUS collaboration [73]. The detail of the data sets will be presented in the next section.

\section{A. H1 data}

The semi-inclusive cross section data for the production of a leading neutron are taken during the years of 2006 and 2007 by the H1 collaboration at HERA in DIS positron-proton scattering [72], which corresponds to an integrate luminosity of $\mathcal{L}=122 \mathrm{pb}^{-1}$, much larger than the previous $\mathrm{H} 1$ measurement [74]. Better experimental capabilities in this measurement lead to the extension of the kinematical coverage of $x$ and $Q^{2}$ to higher values. This leading neutron structure function $F_{2}^{\mathrm{LN}(3)}$, which has been measured by the $\mathrm{H} 1$ experiment at HERA, covers a large range of kinematics of $Q^{2}, \quad 6 \leq Q^{2} \leq 100 \mathrm{GeV}^{2}$, and $x, 1.5 \times 10^{-4} \leq x \leq$ $3 \times 10^{-2}$, for average $y$ values between 0.05 and 0.68 , and the upper limit of the neutron transverse momentum of $p_{T}^{\max }<200 \mathrm{MeV}$. The value of the longitudinal momentum fraction $x_{L}$ covers the range from 0.365 to 0.905 . In order to enhance the relative contribution of a pion exchange $[106,107]$ for these selected DIS events, the value of $p_{T}^{\max }$, which is used for the measurement of $F_{2}^{\mathrm{LN}(3)}$, is set to $200 \mathrm{MeV}$. Considering that the pion exchange mechanism dominates leading neutron production, these data sets can provide constraints on the shape of the pion structure function [108]. In Fig. 1, we plot the nominal coverage of H1 data sets used in our QCD fits. The plot nicely summarizes the universal $\beta, x_{L}$, and $Q^{2}$ dependence of the forward neutron production at HERA.

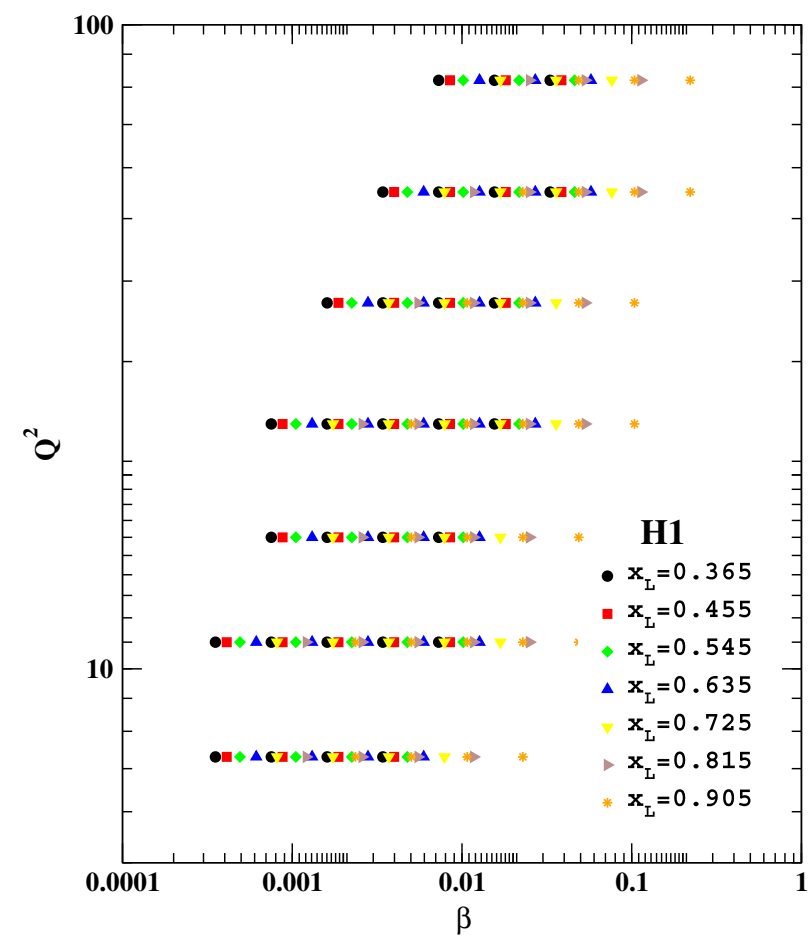

FIG. 1. Nominal coverage of the H1 data sets used in our global QCD fits. The plot nicely summarizes the universal $\beta, x_{L}$, and $Q^{2}$ dependence of the forward neutron production at HERA [72]. For an interpretation of the references to color in the figure legend, the reader is referred to the web version of this article.

\section{B. ZEUS data}

The semi-inclusive cross section for the production of the leading neutron measured by the ZEUS collaboration are also used in our global QCD analysis. The ZEUS collaboration presented the leading neutron production cross sections for $x_{L}>0.2$ in neutral current electron-proton collisions at HERA [73]. Positron and proton energies are $E_{e}=27.5 \mathrm{GeV}$ and $E_{p}=820 \mathrm{GeV}$, respectively, corresponding to a center of mass energy of $\sqrt{s}=300 \mathrm{GeV}$. Similarly to the $\mathrm{H} 1$ experiment, an extensive range of kinematics was covered by the ZEUS data, for $1.1 \times 10^{-4} \leq$ $x \leq 3.2 \times 10^{-2}$ from photoproduction up to a $Q^{2} \sim$ $10^{4} \mathrm{GeV}^{2}$, with $0<y<0.8$, and a neutron scattering angle $\theta_{n}<0.8 \mathrm{mrad}$. The HERA magnet apertures limit the FNC (forward neutron calorimeter) acceptance to a neutron with the production angle less than $\theta_{n}^{\max }=0.8 \mathrm{mrad}$, which corresponds to the transverse momenta of $p_{T}^{\max }=$ $E_{n} \theta_{n}^{\max }=0.656 x_{L} \mathrm{GeV}$. As we already mentioned in the previous section, the distribution of the neutron for the $\mathrm{H} 1$ data is integrated only up to $p_{T}^{\max }=200 \mathrm{MeV}$, so the $\mathrm{H} 1$ and ZEUS data can only be used in the analysis for the longitudinal momentum fraction of $x_{L}=0.3$, which corresponds to $p_{T}^{\max }=197 \mathrm{MeV}$. For higher values of $x_{L}$, the ZEUS data should be scaled to account for the smaller $p_{T}$ range measured by the $\mathrm{H} 1$ collaboration. Of course, in general, we would like to maximize the $\beta, x_{L}$, and $Q^{2}$ 


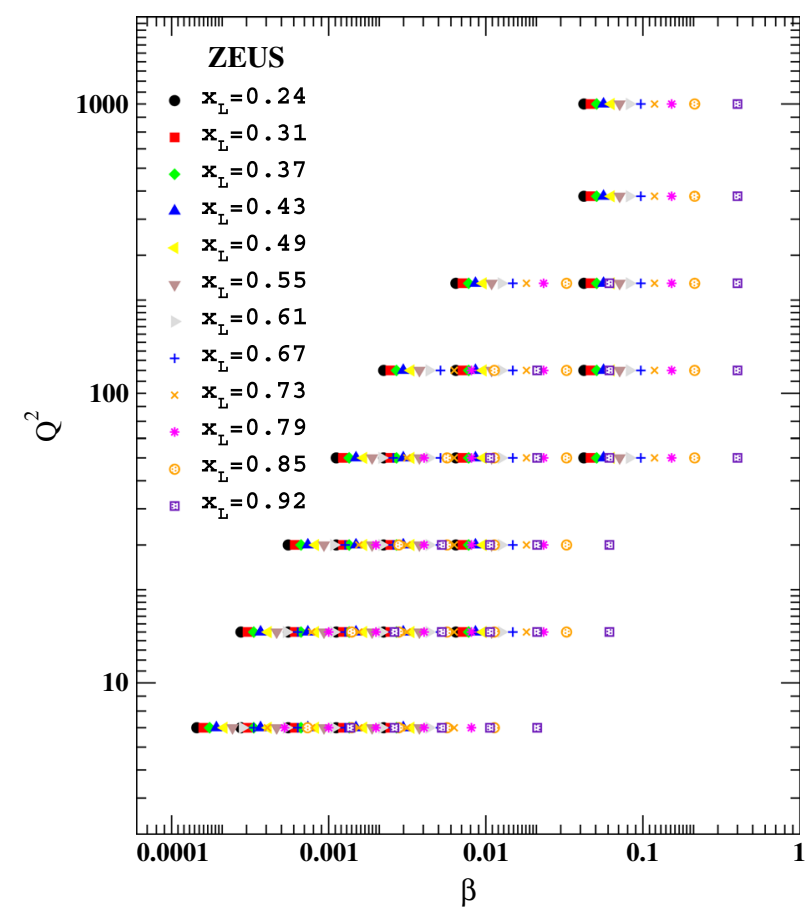

FIG. 2. Nominal coverage of the ZEUS data sets used in our global fits [73]. For an interpretation of the references to color in the figure legend, the reader is referred to the web version of this article.

coverages included in the analysis in order to increase the statistics of our fit. Therefore, we have scaled down the ZEUS data to the $\mathrm{H} 1 p_{T}$ range by using the form of a $p_{T}^{2}$ distribution for the fixed values of $x_{L}$ as [73]

$$
\frac{d \sigma^{\gamma^{*} p \rightarrow X n}}{d p_{T}^{2}} \propto e^{-b\left(x_{L}\right) p_{T}^{2}}
$$

where $\sigma^{\gamma^{*} p \rightarrow X n}$ is the virtual photon-proton cross section for the process $\gamma^{*} p \rightarrow X n$. The slope $b\left(x_{L}\right)$ can be parametrized as $b\left(x_{L}\right)=\left(16.3 x_{L}-4.25\right) \mathrm{GeV}^{-2}$, which is in reasonable accord with the data $[73,81]$. In order to reduce the systematic uncertainties, the ZEUS collaboration measured the neutrontagged cross section $e p \rightarrow e^{\prime} X n$ relative to the inclusive DIS cross section $e p \rightarrow e^{\prime} X$. Considering this ratio as well as a proton structure function, one can obtain the $F_{2}^{\mathrm{LN}(3)}$ values for the various bins of $x, \mathrm{Q}^{2}$, and $y$. The kinematic range of ZEUS forward neutron data are shown in Fig. 2. We should notice here that the $\mathrm{H} 1$ leading neutron data were collected during the 2006-2007 run by an integrated luminosity about 3 times that of the ZEUS data in the DIS region. Consequently, the statistical uncertainties of the $\mathrm{H} 1$ data are much smaller than those for the ZEUS leading neutron spectra.

The measured leading neutron production data points above $Q^{2}=1.0 \mathrm{GeV}^{2}$ used in the SKTJ17 global analysis are listed in Table I. For each data set, we provide the corresponding references, the kinematical coverage of $x_{L}$, $x_{B}$, and $Q^{2}$, the number of data points, and the fitted normalization shifts $\mathcal{N}_{n}$.

\section{THE METHOD OF $\chi^{2}$ MINIMIZATION AND NEUTRON FFS UNCERTAINTIES}

In this section, we outline the details of the SKTJ17 analysis. More specifically, we discuss the selection of data sets, treatment of experimental normalization uncertainties, as well as the determination of the parameters by a global $\chi^{2}$ minimization. We also briefly present the details of the Hessian matrix method for estimating uncertainties. As we noted before, we have performed a careful estimation of the uncertainties using the "Hessian method". An advantage of the Hessian technique is that it allows us to produce sets of eigenvector PDFs, which can be straightforwardly used in computations of other observables, such as the reduced $e^{+} p$ cross section $\sigma_{r}^{\mathrm{LN}(3)}$ as well as the leading neutron structure function $F_{2}^{\mathrm{LN}(3)}$.

\section{A. $\chi^{2}$ minimization}

Global QCD extractions of PDFs, nuclear PDFs as well as polarized PDFs are implemented around an effective $\chi^{2}$ function that quantifies the goodness of the fit to the data for a given set of theoretical parameters, which determine the PDFs at some input scale $\mathrm{Q}_{0}^{2}$. In order to search for optimum PDFs by minimization, the simplest $\chi^{2}$ function is usually given by

$\chi^{2}\left(\left\{p_{i}\right\}\right)=\sum_{n=1}^{N^{\text {exp }}} \sum_{j=1}^{N_{n}^{\text {data }}} w_{j} \frac{\left(\text { Data }_{j}-\text { Theory }_{j}\left(\left\{p_{i}\right\}\right)\right)^{2}}{\delta \text { Data }_{j}}$.

The simple form of $\chi^{2}\left(\left\{p_{i}\right\}\right)$ presented above is appropriate only in the ideal case of data sets with uncorrelated errors. Since most experiments come with additional

TABLE I. A list of all the leading neutron production data points above $Q^{2}=1.0 \mathrm{GeV}^{2}$ used in the SKTJ17 global analysis. For each data set, we provide the corresponding references, the kinematical coverage of $x_{L}, x_{B}$, and $Q^{2}$, the number of data points, and the fitted normalization shifts $\mathcal{N}_{n}$.

\begin{tabular}{|c|c|c|c|c|c|}
\hline Experiments & {$\left[x_{L}^{\min }, x_{L}^{\max }\right]$} & {$\left[x_{B}^{\min }, x_{B}^{\max }\right]$} & $\mathrm{Q}^{2} \mathrm{GeV}^{2}$ & Number of data points & $\mathcal{N}_{n}$ \\
\hline H1 [72] & {$[0.365-0.905]$} & {$\left[1.5 \times 10^{-4}-3.0 \times 10^{-2}\right]$} & $7.3-82$ & 203 & 0.9922 \\
\hline ZEUS [73] & {$[0.240-0.920]$} & {$\left[1.1 \times 10^{-4}-3.2 \times 10^{-2}\right]$} & $7-1000$ & 300 & 1.0033 \\
\hline Total data & & & & 503 & \\
\hline
\end{tabular}


information on the fully correlated normalization uncertainty $\Delta \mathcal{N}_{n}$, Eq. (11) needs to be modified in order to account for such normalization uncertainties. In order to determine the best fit parameters of Eq. (8), we need to minimize the $\chi_{\text {global }}^{2}$ function with the free unknown parameters. $\chi_{\text {global }}^{2}\left(\left\{p_{i}\right\}\right)$ quantifies the goodness of a fit to the data for a set of independent parameters $\left(\left\{p_{i}\right\}\right)$ that specifies the neutron FFs at the input scale $\mathrm{Q}_{0}^{2}=1 \mathrm{GeV}^{2}$. This function is expressed as

$$
\chi_{\text {global }}^{2}\left(\left\{p_{i}\right\}\right)=\sum_{n=1}^{N^{\text {exp }}} w_{n} \chi_{n}^{2},
$$

where $w_{n}$ is a weight factor for the $n$th experiment and

$$
\begin{aligned}
\chi_{n}^{2}\left(\left\{p_{i}\right\}\right)= & \left(\frac{1-\mathcal{N}_{n}}{\Delta \mathcal{N}_{n}}\right)^{2} \\
& +\sum_{j=1}^{N_{n}^{\text {data }}}\left(\frac{\left(\mathcal{N}_{n} \text { Data }_{j}-\text { Theory }_{j}\left(\left\{p_{i}\right\}\right)\right.}{\mathcal{N}_{n} \delta \operatorname{Data}_{j}}\right)^{2},
\end{aligned}
$$

where $N^{\exp }$ correspond to the individual experimental data sets and $N_{n}^{\text {data }}$ correspond to the number of data points in each data set. The normalization factors $\Delta \mathcal{N}_{n}$ in Eq. (13) can be fitted along with the fitted parameters $\left(\left\{p_{i}\right\}\right)$.

TABLE II. The values of $\chi^{2} / N_{\mathrm{pts}}$.. for the data sets included in the SKTJ17 global QCD analysis. A more detailed discussion of the description of the individual data sets and the definitions of $\chi^{2}$ are contained in the text.

\begin{tabular}{lcrr}
\hline \hline Experiment & Data set & \multicolumn{1}{c}{$\chi^{2}$} & $\mathrm{~N}_{\mathrm{pts}}$ \\
\hline H1 & $x_{L}=0.365$ & 24.13 & 29 \\
& $x_{L}=0.455$ & 25.62 & 29 \\
& $x_{L}=0.545$ & 19.36 & 29 \\
& $x_{L}=0.635$ & 19.28 & 29 \\
& $x_{L}=0.725$ & 17.33 & 29 \\
& $x_{L}=0.815$ & 13.23 & 29 \\
All data sets & $x_{L}=0.905$ & 10.15 & 29 \\
ZEUS & & & $\mathbf{1 3 0 . 0 5}$ \\
& & & $\mathbf{2 0 3}$ \\
& $x_{L}=0.240$ & 24.84 & 25 \\
& $x_{L}=0.310$ & 9.68 & 25 \\
& $x_{L}=0.370$ & 11.68 & 25 \\
& $x_{L}=0.430$ & 48.45 & 25 \\
& $x_{L}=0.490$ & 21.11 & 25 \\
& $x_{L}=0.550$ & 24.84 & 25 \\
& $x_{L}=0.610$ & 17.74 & 25 \\
& $x_{L}=0.670$ & 28.18 & 25 \\
& $x_{L}=0.730$ & 6.61 & 25 \\
& $x_{L}=0.790$ & 3.02 & 25 \\
All data sets & $x_{L}=0.850$ & 7.88 & 25 \\
\hline \hline & $x_{L}=0.920$ & 15.02 & 25 \\
& & $\mathbf{2 1 9 . 1 0}$ & $\mathbf{3 0 0}$ \\
\hline
\end{tabular}

The $\chi_{\text {global }}^{2}(\mathrm{p})$ function is minimized by the CERN program library MINUIT [109]. From the $\chi_{\text {global }}^{2}\left(\left\{p_{i}\right\}\right)$ analysis, an error matrix that is the inverse of a Hessian matrix is obtained. In order to determine the sensitivity of the fit to different values of $x_{L}$ collected by H1 and ZEUS collaborations at HERA, we compute the $\chi^{2}$ values for each data set. The data sets included in the SKTJ17 analysis are listed in Table II, together with the $\chi^{2}$ values, defined in Eq. (11), corresponding to each individual data set for each $x_{L}$. This suggests that reasonable fits to the leading neutron cross sections can be obtained within most of the $x_{L}$ values. A more detailed discussion of the description of the individual data sets has been given in Sec. IV.

\section{B. Neutron FFs uncertainties}

As in the case of standard PDFs, the evolved leading neutron fracture functions are linear functions of the input densities. Let $M\left(\beta, Q^{2}, x_{L} ;\left.p_{i}\right|_{i=1} ^{k}\right)$ be the evolved neutron FFs at $Q^{2}$ depending on the parameters $\left.p_{i}\right|_{i=1} ^{k}$. Then its correlated error as given by Gaussian error propagation as [110]

$$
\begin{aligned}
\Delta \beta M\left(\beta, Q^{2}, x_{L}\right)= & \left\{\sum_{i=1}^{k}\left(\frac{\partial \beta M}{\partial p_{i}}\right)^{2} C\left(p_{i}, p_{i}\right)\right. \\
& \left.+\sum_{i \neq j=1}^{k}\left(\frac{\partial \beta M}{\partial p_{i}} \frac{\partial \beta M}{\partial p_{j}}\right) C\left(p_{i}, p_{j}\right)\right\}^{\frac{1}{2}},
\end{aligned}
$$

where $C\left(p_{i}, p_{j}\right)$ are the elements of the covariance matrix obtained in the QCD fit procedure at the input scale $Q_{0}^{2}$. The covariance matrix can be used at any scale of $Q^{2}>Q_{0}^{2}$. The gradients $\partial \beta M / \partial p_{i}$ at this scale can be calculated analytically. Their value at $Q^{2}$ is then calculated by an evolution in $x$ space and are used according to Eq. (14).

In addition to the method presented above, one can also determine the uncertainties of the obtained neutron FFs via the well-known Hessian method and diagonalize the covariance matrix and work in terms of the eigenvectors and eigenvalues. Here, we briefly review the important points for studying the neighborhood of $\chi_{0}^{2}$. The basic procedure is provided in Refs. [35,45,50,111-115].

As we have mentioned earlier, one can find the appropriate parameter set, which minimizes the $\chi_{\text {global }}^{2}$ function. We call this neutron FFs set $S_{0}$. The parameters value of $S_{0}$, i.e. $\left\{p_{1}^{0} \ldots p_{n}^{0}\right\}$, which are extracted from the QCD fit to the H1 and ZEUS leading neutron data, will be presented in Sec. VI. As we will mention latter, we simply fix some of the parameters of our input functional from presented in Eq. (8) at their best-fit values, so that the Hessian matrix only depends on a subset of parameters. 
By moving away the parameters from their obtained values, $\chi^{2}$ increases by the amount of $\Delta \chi^{2}$

$$
\begin{aligned}
\Delta \chi_{\text {global }}^{2} & =\chi_{\text {global }}^{2}(\{p\})-\chi_{0}^{2}\left(\left\{p^{0}\right\}\right) \\
& =\sum_{i, j=1}^{n}\left(p_{i}-p_{i}^{0}\right) H_{i j}\left(p_{j}-p_{j}^{0}\right),
\end{aligned}
$$

where $H_{i j}$ is the Hessian matrix defined as

$$
H_{i j}=\left.\frac{1}{2} \frac{\partial^{2} \chi_{\text {global }}^{2}}{\partial p_{i} \partial p_{j}}\right|_{0}
$$

Now it is convenient to work in terms of the eigenvalues and their corresponding orthogonal eigenvectors of a covariance matrix. It is given by

$$
\sum_{j=1}^{n} C_{i j} v_{j k}=\lambda_{k} v_{i k}
$$

and we should notice here that $C_{i j} \equiv H_{i j}^{-1}$ is the error (or covariance) matrix. The displacement of the parameter $\left\{p_{i}\right\}$ from its obtained minimum $p_{i}^{0}$ can be expressed in terms of the rescaled eigenvectors $e_{i k}=\sqrt{\lambda_{k}} v_{i k}$, that is

$$
p_{i}-p_{i}^{0}=\sum_{k=1}^{n} e_{i k} z_{k}
$$

Considering the orthogonality of the eigenvectors $v_{i k}$ and putting Eq. (18) into Eq. (15), one can write

$$
\Delta \chi_{\text {global }}^{2}=\chi_{\text {global }}^{2}(\{p\})-\chi_{0}^{2}\left(\left\{p^{0}\right\}\right)=\sum_{k=1}^{n} z_{k}^{2} .
$$

The relevant neighborhood of $\chi^{2}$ is the interior of hypersphere with a radius $T$. This means that

$$
\sum_{k=1}^{n} z_{k}^{2} \leq T^{2}
$$

Finally, the neighborhood parameters can be written as

$$
p_{i}\left(s_{k}^{ \pm}\right)=p_{i}^{0} \pm t \sqrt{\lambda_{k}} v_{i k},
$$

with $s_{k}$ as the $k t h$ set of neutron FFs, $t$ adapted to make the desired $T^{2}=\Delta \chi_{\text {global }}^{2}$, which is the tolerance for the required confidence interval (C.L.) and $t=T$ in the quadratic approximation.

Using the method we mentioned above, we accompany the construction of the QCD fit by a reliable estimation of uncertainty. Finally, uncertainties of any observables $\mathcal{O}$, which can be the neutron FFs or reduced cross sections in our case, in the Hessian method can calculate as $[35,114,115]$

$$
\Delta \mathcal{O}=\frac{1}{2}\left[\sum_{k=1}^{n}\left(\mathcal{O}\left(s_{k}^{+}\right)-\mathcal{O}\left(s_{k}^{-}\right)\right)^{2}\right]^{\frac{1}{2}} \text {. }
$$

In the above equation, $\mathcal{O}\left(s_{k}^{+}\right)$and $\mathcal{O}\left(s_{k}^{-}\right)$are the values of $\mathcal{O}$ extracted from the input set of parameters $p_{i}\left(s_{k}^{ \pm}\right)$obtained from Eq. (21). In this paper, we follow the standard Hessian method to calculate the neutron FFs error band as well as the corresponding observables, such as the reduced cross sections. The evolved neutron FFs are attributive functions of the input parameters obtained in the QCD fit procedure at the scale $Q_{0}^{2}$, then their uncertainty can be written applying the standard Hessian method

$$
\Delta \mathcal{O}=\left[\Delta \chi_{\text {global }}^{2} \sum_{i, j=1}^{k} \frac{\partial \mathcal{O}}{\partial p_{i}} C_{i j} \frac{\partial \mathcal{O}}{\partial p_{j}}\right]^{\frac{1}{2}} .
$$

The $\Delta \chi^{2}$ values determine the confidence region, and it is calculated so that the confidence level (C.L.) $P$ becomes the one- $\sigma$-error range $(P=0.68)$ for a given number of parameters $\left(p_{i=N}\right)$ by assuming the normal distribution in the multiparameter space. The neutron FFs are provided with many parameters so that the $\Delta \chi_{\text {global }}^{2}$ value should be calculated.

Assuming correspondence between the confidence level (C.L.) of a normal distribution in multiparameter space and the one of a $\chi^{2}$ distribution with $N$ degrees of freedom, one can define the probability density function as

$$
P_{N}\left(\chi^{2}\right)=\frac{\left(\chi^{2}\right)^{\frac{N}{2}-1}}{2^{\frac{N}{2}} \Gamma\left(\frac{N}{2}\right)} e^{\frac{-\chi^{2}}{2}},
$$

then the confidence level $P$ can be obtained as

$$
\int_{0}^{\Delta \chi^{2}} P_{N}\left(\chi^{2}\right) d \chi^{2}=P(\approx 0.68),
$$

and similarly, for the 90th percentile, we have $P=0.90$. The parameter number in our analysis is eight $(N=8)$, and it leads to $\Delta \chi^{2}=9.27$. The uncertainty of a neutron FFs with respect to the optimized parameters $p_{i=N}$ is then calculated using Eq. (23) by using Hessian matrices and assuming mentioned linear error propagation. For the neutron FFs uncertainty estimation, one can analytically calculate the gradient terms in Eq. (23) at the initial scale $Q_{0}^{2}=1 \mathrm{GeV}^{2}$. For the estimation at an arbitrary $\mathrm{Q}^{2}$, each gradient term is evolved by the DGLAP evolution kernel, and then the neutron FFs uncertainties as well as the uncertainties for any other observables, such as cross sections, are calculated. Here, we calculate the neutron FFs uncertainty with $\Delta \chi^{2}=1$ and 9.27 , which is the most appropriate choice. The Hessian method discussed in the present analysis has been used for estimating TKAA16 NNLO polarized PDFs [38] as well as the KA15 nuclear PDFs analysis [45]. The details of the uncertainty analysis are discussed in detail in Refs. [35,114,115]. 
TABLE III. Parameter values $\left\{p_{i}\right\}$ for the SKTJ17 QCD analysis at the input scale $\mathrm{Q}_{0}^{2}=1 \mathrm{GeV}^{2}$ from the combined $\mathrm{H} 1$ and ZEUS data sets. The values without errors have been fixed after the first minimization since the data do not constrain these unknown parameters well enough. The details of the $\chi^{2}$ analysis and the constraints applied to control the neutron FFs parameters are contained in the text.

\begin{tabular}{lcccc}
\hline \hline Parameters & $\beta M_{\Sigma / P}^{N}\left(\beta, Q_{0}^{2}, x_{L}\right)$ & $p_{i} \pm \delta p_{i}$ & $\beta M_{g / P}^{N}\left(\beta, Q_{0}^{2}, x_{L}\right)$ & $p_{i} \pm \delta p_{i}$ \\
\hline$a$ & $a_{q}$ & $0.116 \pm 0.031$ & $a_{g}$ & $0.0^{*}$ \\
$b$ & $b_{q}$ & $b_{g}$ & $4.884^{*}$ \\
$c$ & $c_{q}$ & $0.260^{*}$ & $c_{g}$ & $9.969^{*}$ \\
$\mathcal{N}$ & $\mathcal{N}_{q}$ & $0.523^{*}$ & $\mathcal{N}_{g}$ & $0.130 \pm 0.027$ \\
$A$ & $A_{q}$ & $0.245 \pm 0.023$ & $A_{g}$ & $0.201^{*}$ \\
$B$ & $B_{q}$ & $B_{g}$ & $1.740 \pm 0.117$ \\
$C$ & $C_{q}$ & $C_{g}$ & $29.865^{*}$ \\
$D$ & $D_{q}$ & $1.430 \pm 0.092$ & $D_{g}$ & $6.733 \pm 0.646$ \\
\hline \hline
\end{tabular}
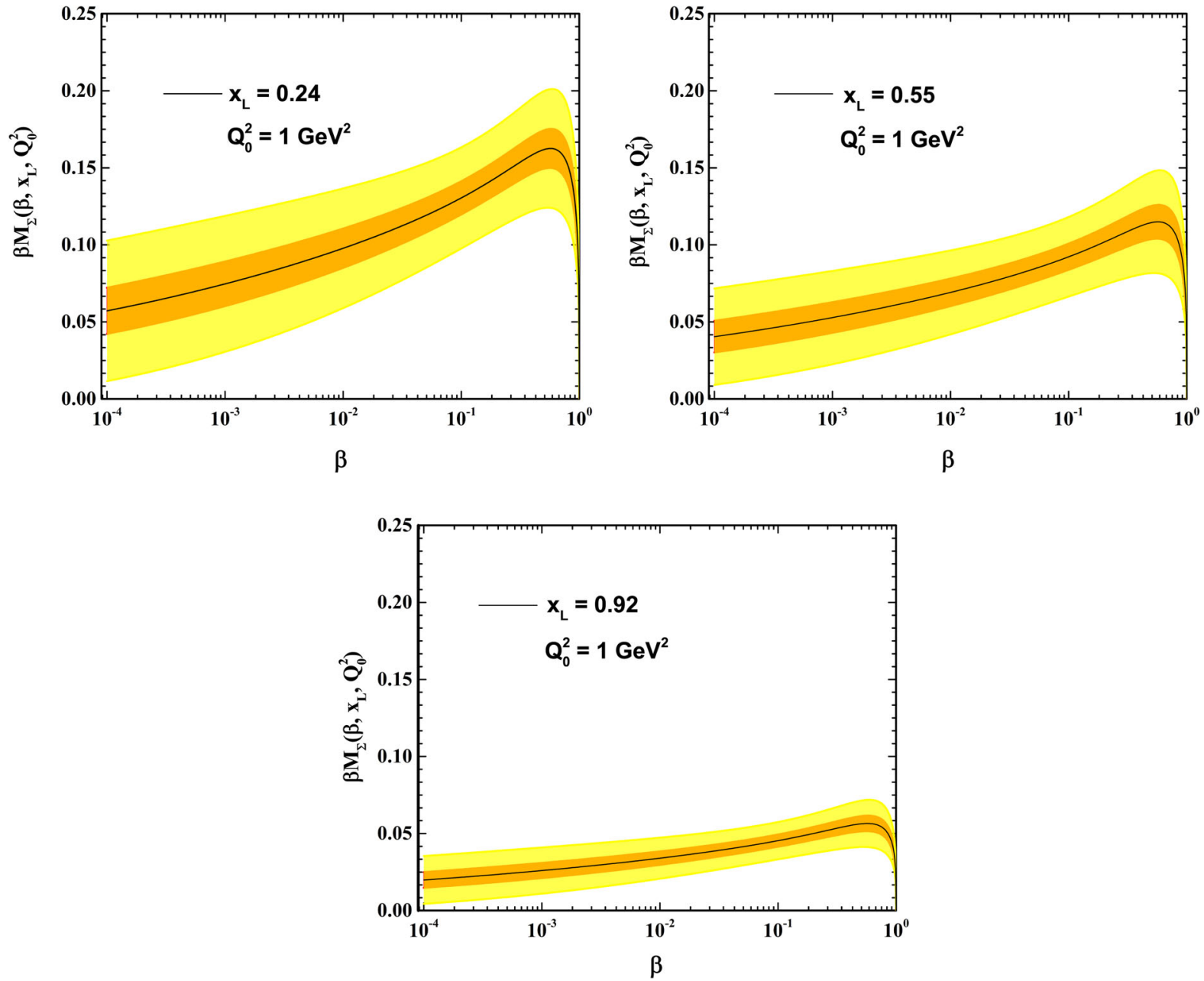

FIG. 3. The singlet momentum distribution as a function of $\beta$ at the input scale $\mathrm{Q}_{0}^{2}=1 \mathrm{GeV}^{2}$ and for three representative bins of $x_{L}=0.24,0.55$, and 0.92 . The error bands are obtained with the Hessian methods (see the text). 


\section{RESULTS AND DISCUSSIONS}

We are in a position to describe the details and all techniques we used for the parametrizations of neutron FFs in the SKTJ17 global analysis. The minimization is carried out with respect to the set of parameters in Eq. (8), $\left\{p_{i}\right\}=\left\{a_{i}, b_{i}, c_{i}, \mathcal{N}_{i}, A_{i}, B_{i}, C_{i}, D_{i}\right\}$. The neutron FFs are evolved to the scales $\mathrm{Q}^{2}>\mathrm{Q}_{0}^{2}$ relevant in the experiment. Like for the case of PDFs parametrization, a particular functional form and the value for $\mathrm{Q}_{0}^{2}$ are not too crucial. The parametrization at the input scale should be flexible enough to accommodate all the DIS data within their ranges of uncertainties. As we mentioned, our input distributions in Eq. (8) follow the standard form used in the fits to the DIS data. In addition to our much more flexible input parametrization presented in Eq. (8), we have repeated our QCD fit with alternative parametrizations, some of them even more flexible than the one we choose. For example, we have also included $\sqrt{x}$ terms, both for the singlet and gluon distributions, even allowing the fit to vary them. We have found no significant improvement in the

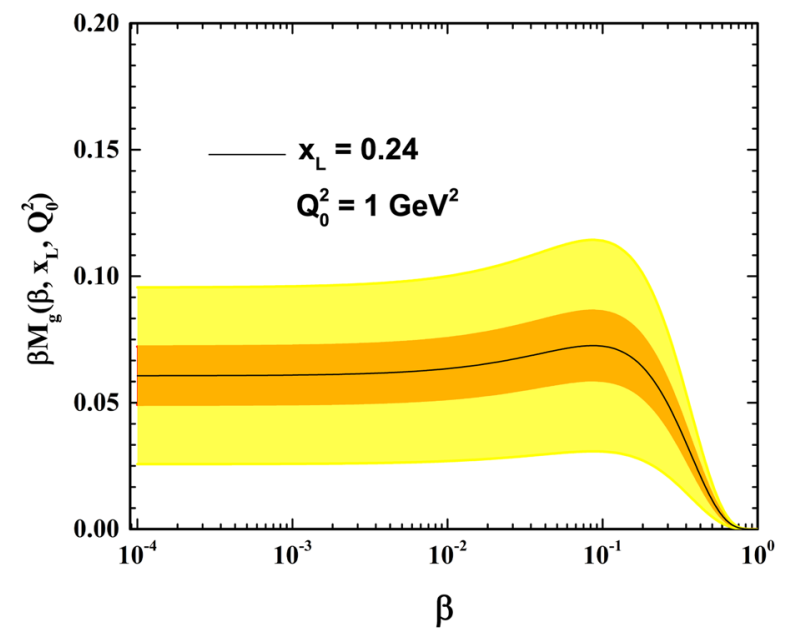

quality of the fit to data or changes of the uncertainty bands. This indicates that the present $\mathrm{H} 1$ and ZEUS leading neutron production data is not really able to discriminate between various forms of the input distributions.

As will be seen from our results presented in this section, we found that our input distributions in Eq. (8) could be considered as good parametrizations to the leading neutron production experimental data.

The parameter values $\left\{p_{i}\right\}$ of the next-to-leading order input neutron FFs at $\mathrm{Q}_{0}^{2}=1 \mathrm{GeV}^{2}$ obtained from the best fit to the combined $\mathrm{H} 1$ and ZEUS leading neutron data sets are presented in Table III.

Parameters marked with $(*)$ are fixed. This is due to the fact that these parameters are only very weakly determined by the fit; consequently, we fixed them to their preferred values. For the sea quark density, we set $A_{q}$ to 0 and for the gluon density, we set $a_{g}$ to 0 in Eq. (8). These only marginally limit the freedom in the functional form. We found that the singlet small- $x_{L}$ coefficient $A_{q}$ as well as the gluon small- $\beta$ coefficient $a_{g}$ are determined with a rather large error and also compatible with zero, so that we fixed

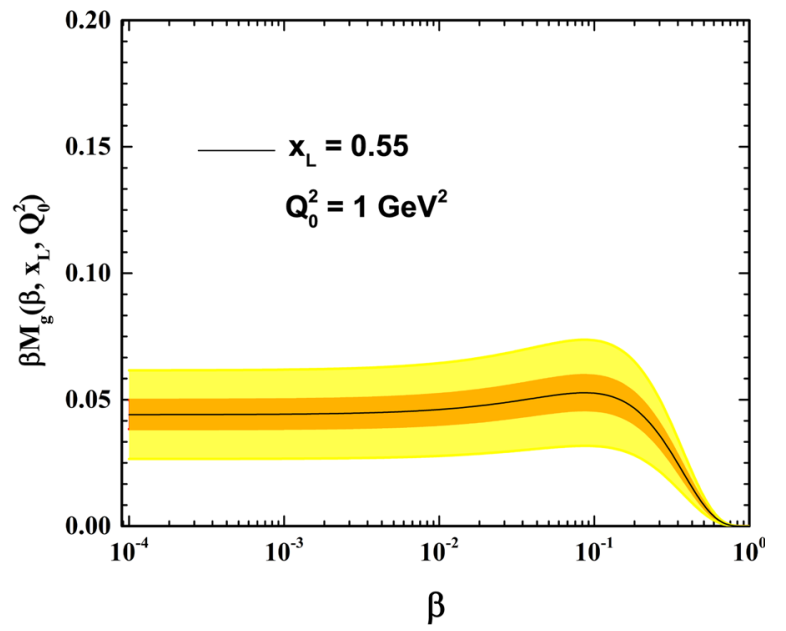

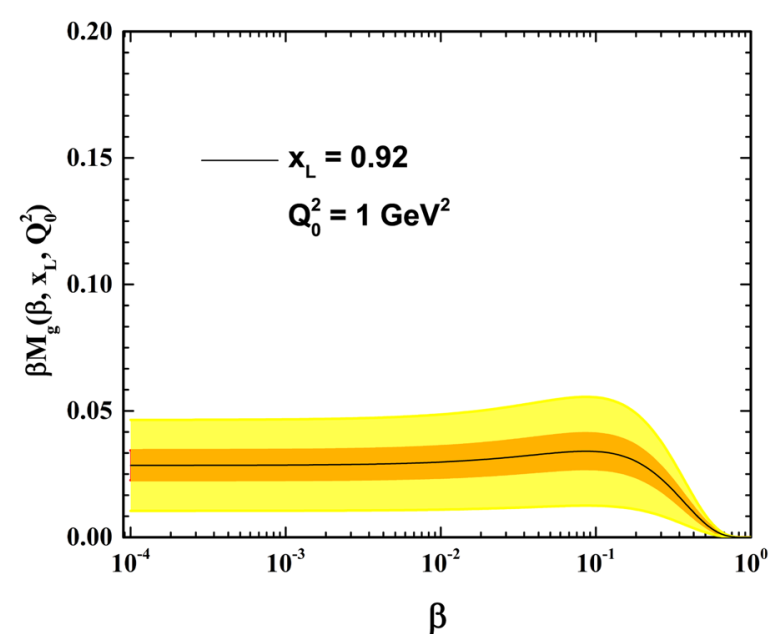

FIG. 4. The gluon momentum distribution as a function of $\beta$ at the input scale $\mathrm{Q}_{0}^{2}=1 \mathrm{GeV}^{2}$ and for three representative bins of $x_{L}=0.24,0.55$, and 0.92. The error bands are obtained with the Hessian methods (see the text). 
them to these values. These are because there are not enough data sensitive to smaller values of $\beta$ and $x_{L}$. Moreover, we found that the factor $\left(1+c_{i} \beta\right)$ in the SKTJ17 parametrization provides flexibility to obtain a good description of the data. Thus, we will make use of the $c_{i}$ coefficients for the sea quark and gluon densities. The parameters $B_{q}$ and $D_{q}$ always came out close to $B_{g}$ and $D_{g}$, so one can set them equal. In order to let enough flexibility to the sea quark and gluon densities, we prefer them to vary differently in the QCD fit. In total, this leaves us with eight free parameters in the SKTJ17 QCD fit (five for sea quarks and three for the gluon density), which we include later on also in our uncertainty estimates. We also tried to relax the imposed constraints discussed above, but found that the
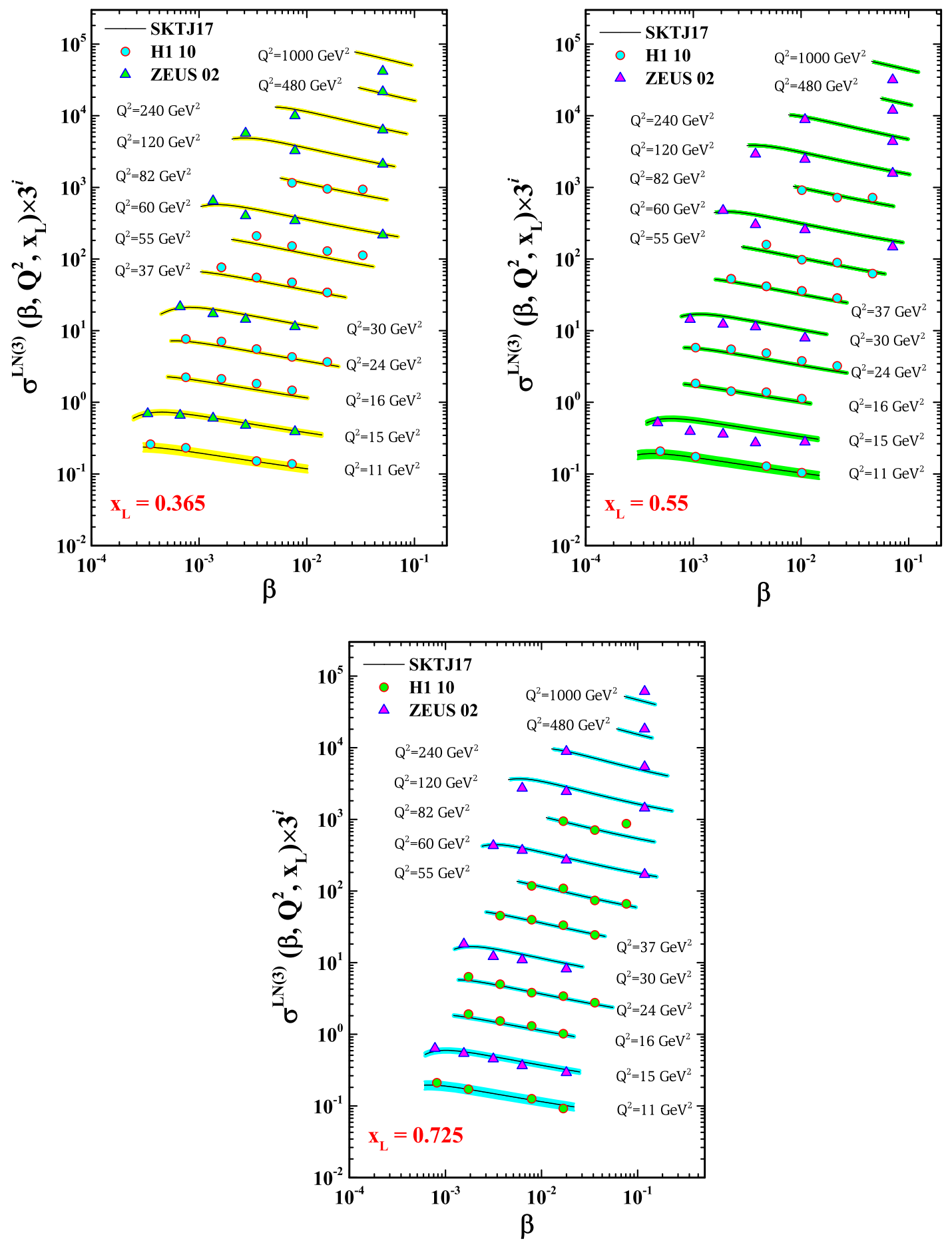

FIG. 5. The reduced cross sections $\sigma_{r}^{\mathrm{LN}(3)}\left(\beta, x_{L}, Q^{2}\right)$ as a function of $\beta$ for some selected values of $\mathrm{Q}^{2}$ (in $\mathrm{GeV}^{2}$ units) and for three representative bins of $x_{L}=0.365,0.550$, and 0.725 . To facilitate the graphical presentation, we have plotted $\sigma_{r}^{\mathrm{LN}(3)}\left(\beta, x_{L}, Q^{2}\right) \times 3^{i}$. 
present leading neutron data are not really sensitive to them. We find $\chi^{2} /$ d.o.f. $=349.16 / 495=0.705$, which yields an acceptable fit to the experimental data.

\section{A. SKTJ17 neutron FFs and their uncertainties}

Our newly obtained singlet and gluon momentum distributions at the input scale $\mathrm{Q}_{0}^{2}=1 \mathrm{GeV}^{2}$ are shown

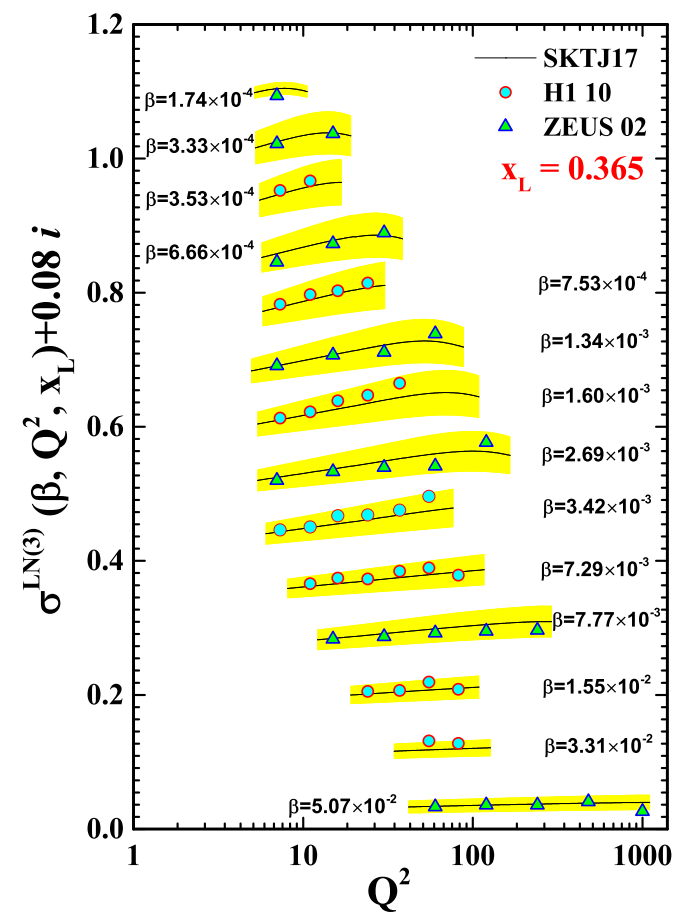

in Figs. 3 and 4 along with estimates of their uncertainties using the Hessian methods for a tolerance of $\Delta \chi^{2}=1$ and 9.27. The results presented for three representative bins of $x_{L}=0.24,0.55$, and 0.92. The inner error band is obtained with the standard "parameter-fitting" criterion, by the choice of a tolerance $\mathrm{T}=\Delta \chi^{2}=1$ for the $68 \%$ (onesigma) confidence level (C.L.) limit while the outer one is

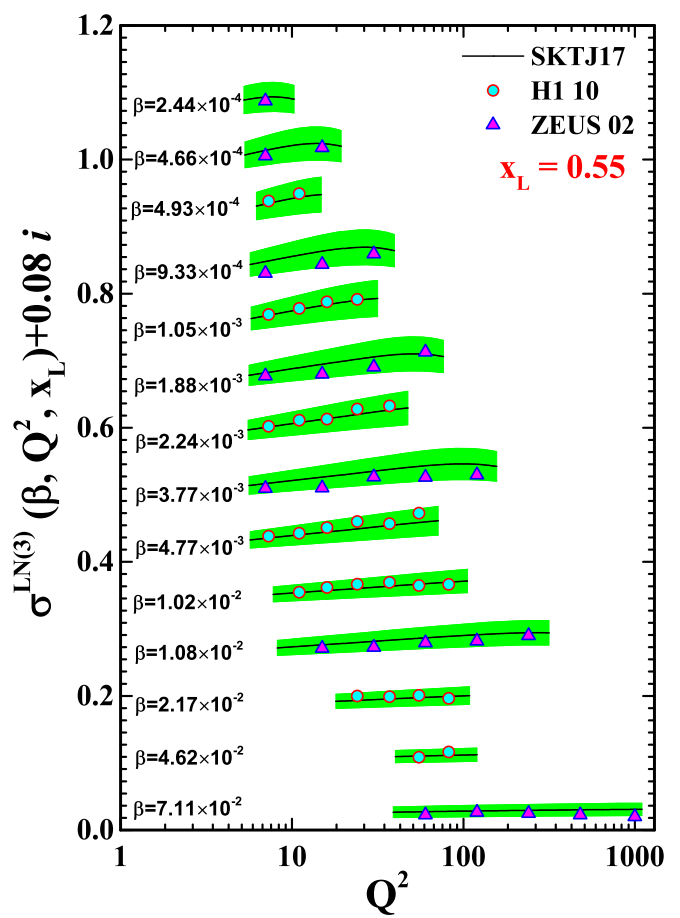

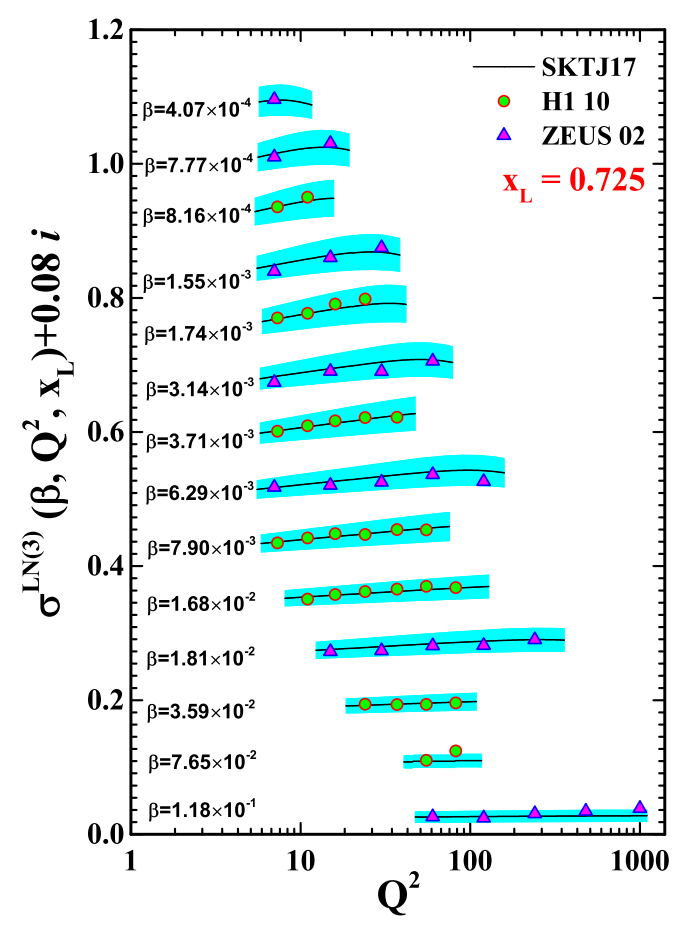

FIG. 6. The reduced cross section $\sigma_{r}^{\mathrm{LN}(3)}\left(\beta, x_{L}, Q^{2}\right)$ as a function of $\mathrm{Q}^{2}$ for some selected values of $\beta$ and for three representative bins of $x_{L}=0.365,0.550$, and 0.725 . To facilitate the graphical presentation, we have plotted $\sigma_{r}^{\operatorname{LN}(3)}\left(\beta, x_{L}, Q^{2}\right)+0.08 i$. 
obtained with the choice of a tolerance $\mathrm{T}=\Delta \chi^{2}=9.27$ using Eq. (25). The main conclusion that can be drawn about the gluon and singlet distributions from the SKTJ17 analysis is that the distributions are important at a large $\beta$. As was stated earlier in Sec. VI, their behavior cannot be precisely determined yet from the available leading neutron production data. In particular, the behavior of the exponent of the $(1-\beta)$ factors in the parametrization, $b_{q}$ and $b_{g}$.

\section{B. Comparison to leading neutron data}

In order to check the reliability of the distributions obtained in our analysis, in the following, we compare results obtained using our best parametrization in Eq. (8) with the leading neutron production data sets presented by the $\mathrm{H} 1$ and ZEUS collaborations, which have been included in the SKTJ17 fit. In Figs. 5, the SKTJ17 theory predictions for the reduced cross section $\sigma_{r}^{\operatorname{LN}(3)}\left(\beta, Q^{2}, x_{L}\right)$ are plotted as a function of $\beta$ for some selected values of $\mathrm{Q}^{2}$. For a better description of the fit quality for a different region of $x_{L}$, three representative bins of $x_{L}=0.365$, 0.55 , and 0.725 are shown. The reduced cross section $\sigma_{r}^{\mathrm{LN}(3)}\left(\beta, Q^{2}, x_{L}\right)$ is scaled by a factor of $3^{i}$ for better visibility in the plots. In order to see the fit quality, the leading neutron production data from the $\mathrm{H} 1$ and ZEUS collaborations $[72,73]$ also added to these plots. From the figures, it is clear that the SKTJ17 QCD fit based on the hard-scattering formula in Eq. (6) together with the neutron FFs initial conditions in Eq. (8) are in acceptable agreement with the H1 and ZEUS data. The plots also show that our results describe the data well, down to the lowest accessible value of $\mathrm{Q}^{2}$ as well as for a different region of $x_{L}$.
In order to study the scale dependence of the H1 and ZEUS leading neutron data, we have plotted the reduced cross sections $\sigma_{r}^{\mathrm{LN}(3)}\left(\beta, Q^{2}, x_{L}\right)$ as a function of $\mathrm{Q}^{2}$ in Fig. 6 for some selected values of $\beta$ and for three representative bins of $x_{L}=0.365,0.550$, and 0.725 . The reduced cross section $\sigma_{r}^{\mathrm{LN}(3)}\left(\beta, Q^{2}, x_{L}\right)$ is scaled by a factor of $0.08 i$ for better visibility in the plots. One can conclude our results show that the scale dependence induced by the evolution equations of Eq. (7) is perfectly consistent with the leading neutron production data. The results clearly show that one can use the fracture functions approach to describe semiinclusive hard processes in perturbative QCD at the kinematic region covered by the electron-proton collider HERA and hadron colliders.

In Fig. 7, our theory predictions for the reduced cross sections $\sigma_{r}^{\mathrm{LN}(3)}\left(\beta, Q^{2}, x_{L}\right)$ are shown as a function of $\beta$. The $\mathrm{H} 1$ (ZEUS) data correspond to $\mathrm{Q}^{2}=7.3(7.0) \mathrm{GeV}^{2}$, and $x_{L}=0.365(0.370)$ in the left panel and $x_{L}=0.725$ (0.730) in the right panel. As shown in the plots, we obtain remarkable agreement with the data in the common $x_{L}$ and $\beta$ range. The plots also clearly show that our approach based on the fracture functions formalism allows a unified description of the leading neutron deep inelastic cross sections.

For completeness, we finally show SKTJ17 theory predictions as a function of $\mathrm{Q}^{2}$ for the reduced cross sections $\sigma_{r}^{\mathrm{LN}(3)}\left(\beta, Q^{2}, x_{L}\right)$ with a representative selection of $\mathrm{H} 1$ and ZEUS data in Fig. 8. In the right panel, the H1 (ZEUS) data correspond to $\beta=7.29(7.77) \times 10^{-3}$ and $x_{L}=0.365(0.370)$. The H1 (ZEUS) data in the left panel correspond to $\beta=1.02(1.08) \times 10^{-2}$ and $x_{L}=$ $0.545(0.550)$. The results demonstrate that SKTJ17 theory
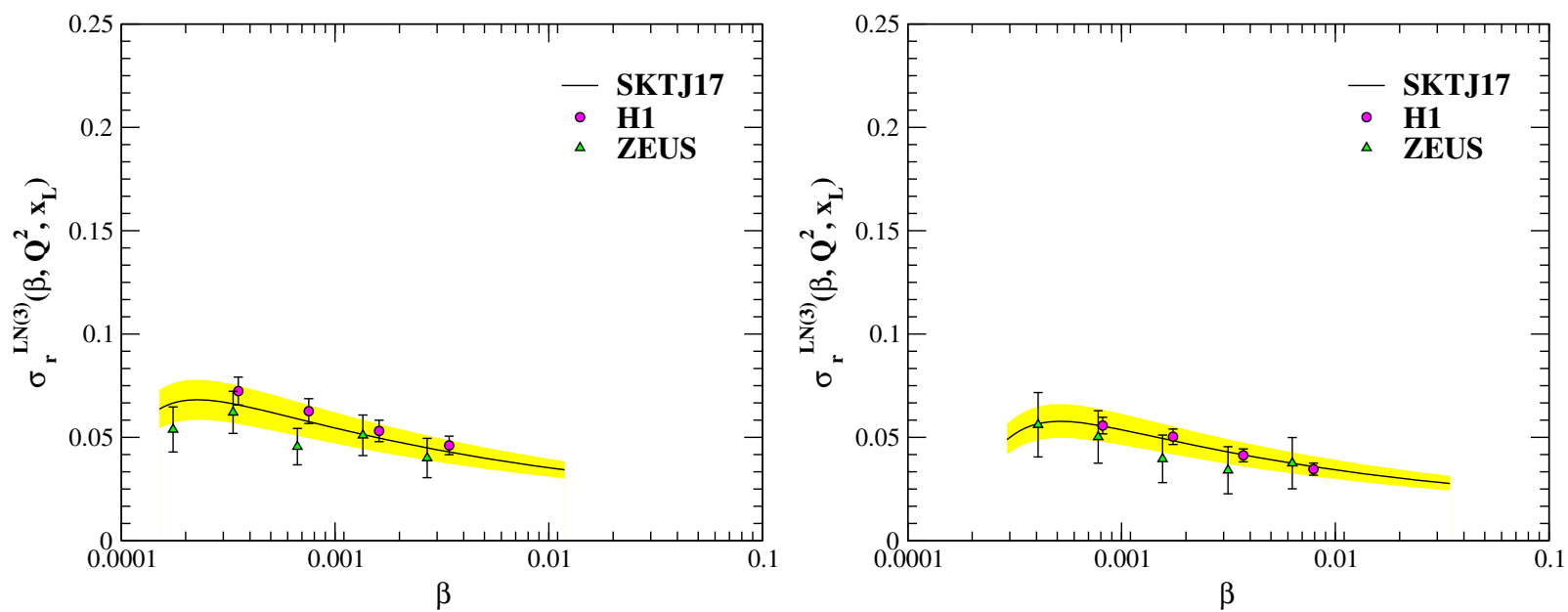

FIG. 7. SKTJ17 theory predictions for the reduced cross sections $\sigma_{r}^{\mathrm{LN}(3)}\left(\beta, Q^{2}, x_{L}\right)$ as a function of $\beta$. The H1 (ZEUS) data correspond to $\mathrm{Q}^{2}=7.3(7.0) \mathrm{GeV}^{2}$, and $x_{L}=0.365(0.370)$ in the left panel and $x_{L}=0.725(0.730)$ in the right panel. The error bars associated with the $\mathrm{H} 1$ and ZEUS data points include systematic and statistical uncertainties, being the total experimental error evaluated in quadrature. 

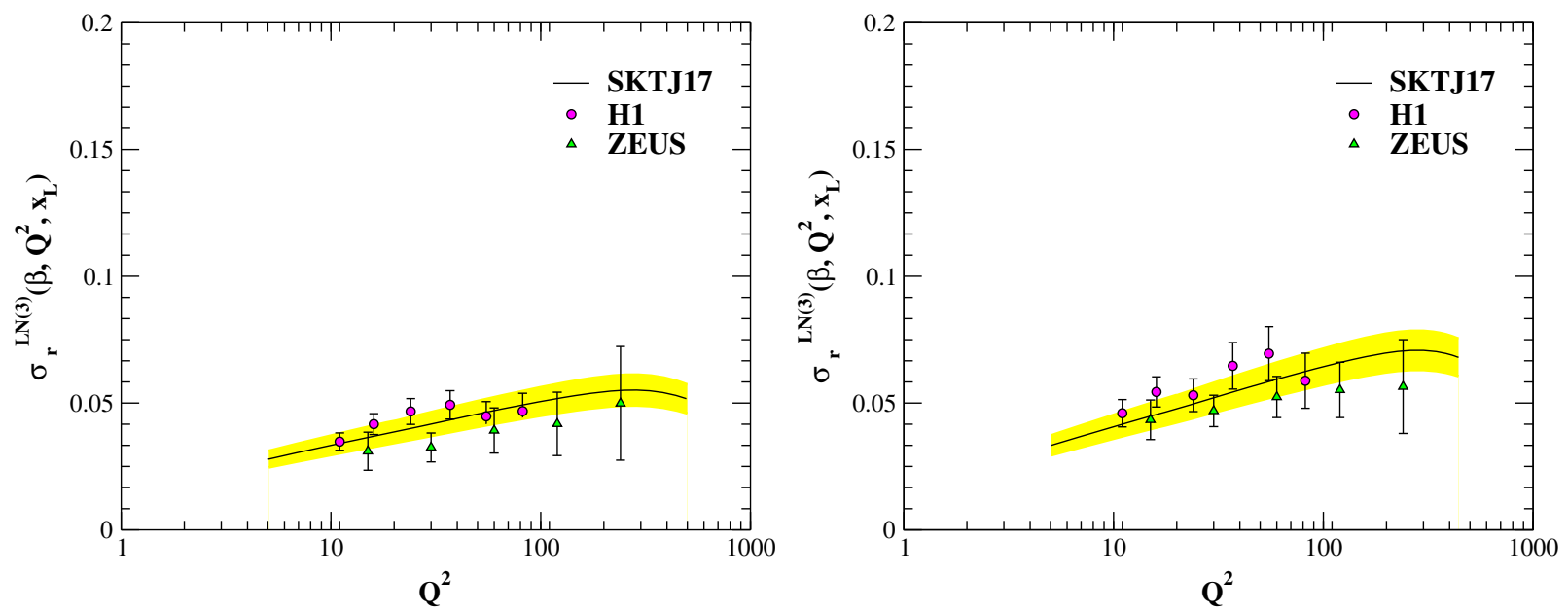

FIG. 8. SKTJ17 theory predictions as a function of $\mathrm{Q}^{2}$ (in $\mathrm{GeV}^{2}$ units). The error bars associated with the H1 and ZEUS data points include systematic and statistical uncertainties, being the total experimental error evaluated in quadrature. In the right panel, the $\mathrm{H} 1$ (ZEUS) data correspond to $\beta=7.29(7.77) \times 10^{-3}$ and $x_{L}=0.365(0.370)$. The H1 (ZEUS) data in the left panel correspond to $\beta=1.02(1.08) \times 10^{-2}$ and $x_{L}=0.545(0.550)$.

predictions can provide a good description of the HERA leading neutron spectra at all kinematics.

In this section, we turned to present our perturbative predictions for the reduced cross section and detail comparison with the available leading neutron production data. Summarizing, our analysis provided a good description of the $\mathrm{H} 1$ and ZEUS data for the leading neutron production in DIS, as a function of $\beta, Q^{2}$, and $x_{L}$. The analysis results presented in this section enabled us to establish the models and parameters that are best able to describe well the existing leading neutron production data from the $\mathrm{H} 1$ and ZEUS collaborations. In spite of the fact that excellent descriptions of the $\mathrm{H} 1$ and ZEUS leading neutron spectra are obtained over the entire range of $\beta, x_{L}$, and $\mathrm{Q}^{2}$ covered by the data, new data could enable further constraints on the extracted neutron FFs. The success of the SKTJ17 global analysis performed here, stands for an explicit check of the pQCD framework in the fracture functions approach for the description of the leading neutron production processes.

\section{LEADING-BARYONS PRODUCTION AT THE LHC}

Let us here conclude by listing some further possible developments of the present framework as well as the experimental efforts. One of the important goals in high energy particle physics is to understand the production of leading-baryons, which have a large fractional longitudinal momentum $x_{L} \geq 0.3$. Recent measurements of leading proton and neutron spectra in electron-proton collisions by the $\mathrm{H} 1$ and ZEUS collaborations at HERA have opened a new window on this subject. Very recently, the H1 collaboration at HERA has measured for the first time, the photoproduction cross section for exclusive $\rho^{0}$ production associated with a leading neutron [116]. Since there is no hard scale presented in exclusive $\rho^{0}$ production, one can use a phenomenological approach such as the Regge theory or color dipole formalism, to describe these kind of reactions [117-119].

Nowadays, our understanding of the hadron structure as well as the QCD dynamics have advanced with the successful operation and precise data at the HERA collider. In addition to the HERA collider, the next generation of high energy and high luminosity electron-proton colliders, such as the Large Hadron Electron Collider (LHeC) [120-122] as well as the Future Circular HadronElectron Collider (FCC-he) [123], which is proposed to build on the same site with LHC, could help to study the leading-baryon processes.

Another possibility is the use of the hadronic colliders. One of the important issues which have strong implications in the forward physics at hadron colliders, is the understanding of the leading neutron processes. A very rich program at the Large Hadron Collider (LHC) is being pursued in forward physics with sufficient experimental information [124,125]. Finally, the upcoming experiment at Jefferson Lab (JLAB) plans to take data on the production of the leading protons in the $e n \rightarrow e p X$ process [126-128]. With the help of more and precise upcoming experimental data on such processes, a new era for the theoretical understanding of strong interactions in the soft, nonperturbative regime will be open [126].

\section{SUMMARY AND CONCLUSION}

In the recent years, several dedicated experiments at the electron-proton collider HERA have collected high-precision data on the spectrum of leading-baryons carrying a large fraction of the proton's energy. However, the experimental information on the leading-baryons 
production in lepton DIS, $e p \rightarrow e^{\prime} B X$, is still rather scarce. In addition to these experimental efforts, much successful phenomenology has been developed in understanding the mechanism of leading-baryon productions. The presence of a leading baryon in the final state of lepton DIS provides valuable information on the relationship between the soft and hard aspects of the strong interaction.

In this work, we have presented the SKTJ17 NLO QCD analysis of neutron FFs using available and up-to-date data from the forward neutron production at HERA [72,73]. It is shown that an approach based on the fracture functions formalism allows us to phenomenologically parametrize the neutron FFs. We also have shown that a standard simple parametric form for this function gives a very accurate description of the available leading neutron production data. Finally, one can conclude that our obtained results based on the fracture function approach agree well with the scale dependence of the leading neutron production data. Completing such a picture is crucial as hadron colliders enter an era of a new generation of experimental data capable of testing this formalism. In order to asses the uncertainties in the resulting neutron FFs and the corresponding observables, associated with the uncertainties in the data, we have made extensive use of the Hessian method.

A FORTRAN package containing SKTJ17 neutron FFs parametrization as well as the corresponding error set can be obtained via email from the authors upon request. This FORTRAN package also includes an example program to illustrate the use of the routines.

\section{ACKNOWLEDGMENTS}

The authors are especially grateful Garry Levman, Katja Kruger, Stefan Schmitt, Mohsen Khakzad from the H1 and ZEUS collaborations for many useful discussions and comments. We are also thankful to Federico Ceccopieri, Fatemeh Arbabifar, Muhammad Goharipour, and S. Atashbar Tehrani for numerous informative discussions. H. K. is indebted the University of Science and Technology of Mazandaran and the School of Particles and Accelerators, Institute for Research in Fundamental Sciences (IPM), for financial support of this project. F. T.-S. and K. J. also acknowledge the Ferdowsi University of Mashhad.
[1] H. Abramowicz et al. (ZEUS Collaboration), Phys. Rev. D 93, 092002 (2016).

[2] I. Abt, A. M. Cooper-Sarkar, B. Foster, C. Gwenlan, V. Myronenko, O. Turkot, and K. Wichmann, Phys. Rev. D 94, 052007 (2016).

[3] H. Abramowicz et al. (H1 and ZEUS Collaborations), Eur. Phys. J. C 75, 580 (2015).

[4] H. Abramowicz et al. (ZEUS Collaboration), Phys. Rev. D 90, 072002 (2014).

[5] V. Andreev et al. (H1 Collaboration), Eur. Phys. J. C 74, 2814 (2014).

[6] F. D. Aaron et al. (H1 and ZEUS Collaborations), J. High Energy Phys. 01 (2010) 109.

[7] F. D. Aaron et al. (H1 Collaboration), Eur. Phys. J. C 64, 561 (2009).

[8] F. D. Aaron et al. (H1 Collaboration), Eur. Phys. J. C 63, 625 (2009).

[9] Tevatron Electroweak Working Group Collaboration et al. (CD and D0 Collaboration), arXiv:1608.01881.

[10] V. M. Abazov et al. (D0 Collaboration), Phys. Rev. D 95, 031101 (2017).

[11] V. M. Abazov et al. (D0 Collaboration), Phys. Rev. D 95, 011101 (2017).

[12] T. A. Aaltonen et al. (CDF Collaboration), Phys. Rev. D 94, 032008 (2016).

[13] V. M. Abazov et al. (D0 Collaboration), Phys. Rev. D 94, 032004 (2016).

[14] T. Aaltonen et al. (CDF Collaboration), Phys. Rev. D 78, 052006 (2008); 79, 119902(E) (2009).
[15] A. Abulencia et al. (CDF Collaboration), Phys. Rev. D 74, 071103 (2006).

[16] V. M. Abazov et al. (D0 Collaboration), Phys. Rev. Lett. 101, 062001 (2008).

[17] A. Abulencia et al. (CDF Collaboration), Phys. Rev. D 75, 092006 (2007); 75, 119901(E) (2007).

[18] B. Abbott et al. (D0 Collaboration), Phys. Rev. Lett. 86, 1707 (2001).

[19] CMS Collaboration, Report No. CMS-PAS-SMP-16-008.

[20] A. M. Sirunyan et al. (CMS Collaboration), arXiv:1703 .01630 .

[21] G. Aad et al. (ATLAS Collaboration), Eur. Phys. J. C 73, 2509 (2013).

[22] G. Aad et al. (ATLAS Collaboration), Phys. Rev. D 86, 014022 (2012).

[23] S. Chatrchyan et al. (CMS Collaboration), J. High Energy Phys. 12 (2013) 030.

[24] S. Chatrchyan et al. (CMS Collaboration), Phys. Rev. D 85, 032002 (2012).

[25] G. Aad et al. (ATLAS Collaboration), Phys. Lett. B 725, 223 (2013).

[26] R. Aaij et al. (LHCb Collaboration), J. High Energy Phys. 02 (2013) 106.

[27] R. Aaij et al. (LHCb Collaboration), J. High Energy Phys. 05 (2015) 109.

[28] S. Chatrchyan et al. (CMS Collaboration), J. High Energy Phys. 04 (2011) 050.

[29] G. Aad et al. (ATLAS Collaboration), Phys. Rev. D 85, 072004 (2012). 
[30] S. Chatrchyan et al. (CMS Collaboration), Phys. Rev. Lett. 109, 111806 (2012).

[31] C. Bourrely and J. Soffer, Nucl. Phys. A941, 307 (2015).

[32] R. D. Ball et al., Nucl. Phys. B867, 244 (2013).

[33] L. A. Harland-Lang, A. D. Martin, P. Motylinski, and R. S. Thorne, Eur. Phys. J. C 75, 204 (2015).

[34] R. D. Ball et al. (NNPDF Collaboration), J. High Energy Phys. 04 (2015) 040.

[35] A. D. Martin, W. J. Stirling, R. S. Thorne, and G. Watt, Eur. Phys. J. C 63, 189 (2009).

[36] J. Gao, M. Guzzi, J. Huston, H.-L. Lai, Z. Li, P. Nadolsky, J. Pumplin, D. Stump, and C.-P. Yuan, Phys. Rev. D 89, 033009 (2014).

[37] S. Alekhin, J. Blumlein, and S. Moch, Phys. Rev. D 86, 054009 (2012).

[38] F. Taghavi-Shahri, H. Khanpour, S. Atashbar Tehrani, and Z. Alizadeh Yazdi, Phys. Rev. D 93, 114024 (2016).

[39] R. D. Ball, S. Forte, A. Guffanti, E. R. Nocera, G. Ridolfi, and J. Rojo, Nucl. Phys. B874, 36 (2013).

[40] P. Jimenez-Delgado, H. Avakian, and W. Melnitchouk, Phys. Lett. B 738, 263 (2014).

[41] N. Sato, W. Melnitchouk, S. E. Kuhn, J. J. Ethier, and A. Accardi, Phys. Rev. D 93, 074005 (2016).

[42] E. R. Nocera, Phys. Lett. B 742, 117 (2015).

[43] E. Leader, A. V. Sidorov, and D. B. Stamenov, Phys. Rev. D 91, 054017 (2015).

[44] E. R. Nocera, R. D. Ball, S. Forte, G. Ridolfi, and J. Rojo, Nucl. Phys. B887, 276 (2014).

[45] H. Khanpour and S. Atashbar Tehrani, Phys. Rev. D 93, 014026 (2016).

[46] K. J. Eskola, P. Paakkinen, H. Paukkunen, and C. A. Salgado, Eur. Phys. J. C 77, 163 (2017).

[47] K. Kovarik et al., Phys. Rev. D 93, 085037 (2016).

[48] M. Klasen, K. Kovarik, and J. Potthoff, arXiv:1703.02864.

[49] R. Wang, X. Chen, and Q. Fu, arXiv:1611.03670.

[50] D. de Florian, R. Sassot, P. Zurita, and M. Stratmann, Phys. Rev. D 85, 074028 (2012).

[51] M. Goharipour and H. Mehraban, Adv. High Energy Phys. 2017, 3802381 (2017).

[52] C. E. Carlson and M. Freid, arXiv:1702.05775.

[53] A. Accardi, W. Melnitchouk, J. F. Owens, M. E. Christy, C. E. Keppel, L. Zhu, and J. G. Morfin, Phys. Rev. D 84, 014008 (2011).

[54] J. F. Owens, A. Accardi, and W. Melnitchouk, Phys. Rev. D 87, 094012 (2013).

[55] C. Monahan and K. Orginos, J. High Energy Phys. 03 (2017) 116.

[56] H. Dahiya and M. Randhawa, Phys. Rev. D 93, 114030 (2016).

[57] P. Jimenez-Delgado, A. Accardi, and W. Melnitchouk, Phys. Rev. D 89, 034025 (2014).

[58] E. R. Nocera and E. Santopinto, arXiv:1611.07980.

[59] R. D. Ball, E. R. Nocera, and J. Rojo, Eur. Phys. J. C 76, 383 (2016).

[60] M. Goharipour and H. Mehraban, Phys. Rev. D 95, 054002 (2017).

[61] P. Ru, S. A. Kulagin, R. Petti, and B. W. Zhang, Phys. Rev. D 94, 113013 (2016).

[62] H. Haider, F. Zaidi, M. Sajjad Athar, S. K. Singh, and I. Ruiz Simo, Nucl. Phys. A955, 58 (2016).
[63] A. Accardi, L. T. Brady, W. Melnitchouk, J. F. Owens, and N. Sato, Phys. Rev. D 93, 114017 (2016).

[64] N. Armesto, H. Paukkunen, J. M. Penín, C. A. Salgado, and P. Zurita, Eur. Phys. J. C 76, 218 (2016).

[65] L. Frankfurt, V. Guzey, M. Strikman, and M. Zhalov, Phys. Lett. B 752, 51 (2016).

[66] V. Guzey, E. Kryshen, M. Strikman, and M. Zhalov, Phys. Lett. B 726, 290 (2013).

[67] L. Frankfurt, V. Guzey, and M. Strikman, arXiv:1612 .08273 .

[68] V. Guzey, M. Strikman, and M. Zhalov, Phys. Rev. C 95, 025204 (2017).

[69] A. Accardi, V. Guzey, A. Prokudin, and C. Weiss, Eur. Phys. J. A 48, 92 (2012).

[70] L. Frankfurt, V. Guzey, and M. Strikman, Phys. Rep. 512, 255 (2012).

[71] S. Alekhin, J. Blümlein, S. Moch, and R. Placakyte, arXiv:1701.05838.

[72] F. D. Aaron et al. (H1 Collaboration), Eur. Phys. J. C 68, 381 (2010).

[73] S. Chekanov et al. (ZEUS Collaboration), Nucl. Phys. B637, 3 (2002).

[74] C. Adloff et al. (H1 Collaboration), Eur. Phys. J. C 6, 587 (1999).

[75] J. Breitweg et al. (ZEUS Collaboration), Nucl. Phys. B596, 3 (2001).

[76] S. Chekanov et al. (ZEUS Collaboration), Phys. Lett. B 610, 199 (2005).

[77] A. Aktas et al. (H1 Collaboration), Eur. Phys. J. C 41, 273 (2005).

[78] S. Chekanov et al. (ZEUS Collaboration), Phys. Lett. B 590, 143 (2004).

[79] S. Chekanov et al. (ZEUS Collaboration), Nucl. Phys. B776, 1 (2007).

[80] S. Chekanov et al. (ZEUS Collaboration), J. High Energy Phys. 06 (2009) 074.

[81] S. Chekanov et al. (ZEUS Collaboration), Nucl. Phys. B658, 3 (2003).

[82] V. Andreev et al. (H1 Collaboration), Eur. Phys. J. C 74, 2915 (2014).

[83] L. Trentadue and G. Veneziano, Phys. Lett. B 323, 201 (1994).

[84] F. A. Ceccopieri and L. Trentadue, Phys. Lett. B 655, 15 (2007).

[85] A. Szczurek, N. N. Nikolaev, and J. Speth, Phys. Lett. B 428, 383 (1998).

[86] F. A. Ceccopieri, Eur. Phys. J. C 74, 3029 (2014).

[87] D. de Florian and R. Sassot, Phys. Rev. D 58, 054003 (1998).

[88] D. de Florian and R. Sassot, Phys. Rev. D 56, 426 (1997).

[89] J. A. M. Vermaseren, A. Vogt, and S. Moch, Nucl. Phys. B724, 3 (2005).

[90] Y. L. Dokshitzer, Zh. Eksp. Teor. Fiz. 73, 1216 (1977) [Sov. Phys. JETP 46, 641 (1977)].

[91] V. N. Gribov and L. N. Lipatov, Yad. Fiz. 15, 781 (1972) [Sov. J. Nucl. Phys. 15, 438 (1972)].

[92] L. N. Lipatov, Yad. Fiz. 20, 181 (1974) [Sov. J. Nucl. Phys. 20, 94 (1975)].

[93] G. Altarelli and G. Parisi, Nucl. Phys. B126, 298 (1977).

[94] G. Camici, M. Grazzini, and L. Trentadue, Phys. Lett. B 439, 382 (1998). 
[95] A. Daleo, C. A. Garcia Canal, and R. Sassot, Nucl. Phys. B662, 334 (2003).

[96] A. Daleo and R. Sassot, Nucl. Phys. B673, 357 (2003).

[97] L. Trentadue, Nucl. Phys. B, Proc. Suppl. 54A, 176 (1997).

[98] L. Trentadue, Nucl. Phys. B, Proc. Suppl. 39, 50 (1995).

[99] A. Vogt, S. Moch, and J. A. M. Vermaseren, Nucl. Phys. B691, 129 (2004).

[100] S. Moch, J. A. M. Vermaseren, and A. Vogt, Nucl. Phys. B688, 101 (2004).

[101] W. L. van Neerven and A. Vogt, Nucl. Phys. B588, 345 (2000).

[102] W. L. van Neerven and A. Vogt, Nucl. Phys. B568, 263 (2000).

[103] S. Moch, J. A. M. Vermaseren, and A. Vogt, Nucl. Phys. B889, 351 (2014).

[104] C. Patrignani et al. (Particle Data Group Collaboration), Chin. Phys. C 40, 100001 (2016).

[105] K. A. Olive et al. (Particle Data Group Collaboration), Chin. Phys. C 38, 090001 (2014).

[106] H. Holtmann, G. Levman, N. N. Nikolaev, A. Szczurek, and J. Speth, Phys. Lett. B 338, 363 (1994).

[107] B. Kopeliovich, B. Povh, and I. Potashnikova, Z. Phys. C 73, 125 (1996).

[108] J. R. McKenney, N. Sato, W. Melnitchouk, and C. R. Ji, Phys. Rev. D 93, 054011 (2016).

[109] F. James and M. Roos, Comput. Phys. Commun. 10, 343 (1975).

[110] J. Blumlein and H. Bottcher, Nucl. Phys. B636, 225 (2002).

[111] T. J. Hou et al., J. High Energy Phys. 1703 (2017) 099.

[112] S. M. Moosavi Nejad, H. Khanpour, S. Atashbar Tehrani, and M. Mahdavi, Phys. Rev. C 94, 045201 (2016).

[113] H. Khanpour, A. Mirjalili, and S. Atashbar Tehrani, Phys. Rev. C 95, 035201 (2017).
[114] A. D. Martin, R. G. Roberts, W. J. Stirling, and R. S. Thorne, Eur. Phys. J. C 28, 455 (2003).

[115] J. Pumplin, D. Stump, R. Brock, D. Casey, J. Huston, J. Kalk, H. L. Lai, and W. K. Tung, Phys. Rev. D 65, 014013 (2001).

[116] V. Andreev et al. (H1 Collaboration), Eur. Phys. J. C 76, 41 (2016).

[117] M. B. G. Ducati, F. Kopp, M. V. T. Machado, and S. Martins, Phys. Rev. D 94, 094023 (2016).

[118] P. Lebiedowicz, O. Nachtmann, and A. Szczurek, Phys. Rev. D 95, 034036 (2017).

[119] V. P. Goncalves, F. S. Navarra, and D. Spiering, Phys. Rev. D 93, 054025 (2016).

[120] J. L. Abelleira Fernandez et al., arXiv:1211.4831.

[121] J. L. Abelleira Fernandez et al. (LHeC Study Group Collaboration), arXiv:1211.5102.

[122] Y. C. Acar, U. Kaya, B. B. Oner, and S. Sultansoy, arXiv: 1510.08284.

[123] Y. C. Acar, A. N. Akay, S. Beser, H. Karadeniz, U. Kaya, B. B. Oner, and S. Sultansoy, arXiv:1608.02190.

[124] K. Akiba et al. (LHC Forward Physics Working Group Collaboration), J. Phys. G 43, 110201 (2016).

[125] M. Albrow et al. (CMS and TOTEM diffractive and forward physics working Group Collaboration), Reports No. CERN-CMS-Note-2007-002, No. CERN-LHCC2006-039-G-124, No. CMS-Note-2007-002, No. TOTEM-Note-2006-005, No. LHCC-G-124, No. CERN-TOTEM-Note-2006-005.

[126] V. P. Goncalves, B. D. Moreira, F. S. Navarra, and D. Spiering, Phys. Rev. D 94, 014009 (2016).

[127] J. Annand, D. Dutta, C. Keppel, P. King, and B. Wojtsekhowski, Jefferson Lab experiment Report No. PR12-15-006.

[128] R. A. Montgomery et al., AIP Conf. Proc. 1819, 030004 (2017). 